\title{
Suspect and non-target screening for contaminants of emerging concern in an urban estuary
}

Zhenyu Tian ${ }^{1,2 *}$, Katherine T. Peter ${ }^{1,2}$, Alex D. Gipe ${ }^{1,2}$, Haoqi Zhao ${ }^{3}$, Fan $\mathrm{Hou}^{3}$, David A. Wark $^{1,2}$, Tarang Khangaonkar ${ }^{4}$, Edward P. Kolodziej ${ }^{1,2,3}$, C. Andrew James ${ }^{1,2 *}$

${ }^{1}$ Center for Urban Waters, Tacoma, WA, 98421, USA

${ }^{2}$ Interdisciplinary Arts and Sciences, University of Washington Tacoma, Tacoma, WA, 98421, USA

${ }^{3}$ Department of Civil and Environmental Engineering, University of Washington, Seattle, WA, 98195, USA

${ }^{4}$ Pacific Northwest National Laboratories, 1100 Dexter Avenue N, Seattle, WA 98011, USA

*Corresponding author contact information

Email: tianzy@uw.edu,jamesca@uw.edu

\section{Supporting information}

Pages: 20

Tables: 8

Figure: 6 


\section{List of reference standards}

Most reference standards for identification and semi-quantification were purchased from SigmaAldrich (St. Louis, MO, USA). The name, purity, and CAS registration number were listed below: triclopyr (98\%, CAS 55335-06-3), 2,4-dinitrophenol (98\%, CAS 51-28-5), chlorothalonil (98\%,

CAS 28343-61-5), bisphenol S (98\%, CAS 80-09-1), venlafaxine hydrochloride (98\%, CAS 99300-78-4), O-desvenlafaxine (93413-62-8 ), lidocaine (CAS 137-58-6), tebuthiuron (34014-181), 2,6-dichlorobenzamide $(97 \%, 2008-58-4)$, sucralose (98\%, 56038-13-2), 4-nitrophenol (10002-7), hexa(methoxymethyl)melamine (HMMM; 95\%, CAS 3089-11-0), 1,3-diphenylguanidine (97\%, CAS 102-06-7), 1,3-dicyclohexylurea (98\%, CAS 2387-23-7), N-butylbenzenesulfonate (99\%, CAS 3622-84-2), tris(2-chloroethyl)phosphate (95\%, CAS 115-96-8), tris(2butoxyethyl)phosphate ( 95\%, CAS 78-51-3), fluridone (CAS 59756-60-4), diuron (CAS 330-541), diisononyl phthalate (99\%, CAS 28553-12-0), 1-cyclohexyl-2-pyrrolidone (99\%, CAS 683724-7), 5-methyl-1-H-benzotriazole (98\%, CAS 136-85-6), N,N-dimethyldodecylamine (99\% CAS 1643-20-5), 2-benzothiazolesulfonic acid potassium salt (CAS 941-57-1), salicylic acid (CAS 6972-7), 4-hydroxyquinoline (98\%, CAS 611-36-9), 2-mercaptobenzothiazole (97\%, CAS 149-304), benzotriazole (98\%, CAS 95-14-7), triisopropanolamine (95\%, CAS 122-20-3), caffeine (CAS 58-08-2), propamocarb (CAS 24579-73-5), metsulfuron-methyl (CAS 74223-64-6), rimsulfuron (CAS 122931-48-0), vanillin (CAS 121-33-5), metoprolol tartrate (98\%, CAS 56392-17-7). Methamphetamine (CAS 7632-10-2) was purchased from Cerilliant (98\%, Round Rock, TX, USA 78665). PFOS (CAS 1763-23-1), PFHxS (CAS 355-46-4), PFBS (CAS 375-73-5), PFOA (CAS 335-67-1), PFHpA (CAS 375-85-9), PFHxA (CAS 307-24-4), PFPeA (CAS 2706-90-3) were purchased from Wellington Laboratories (Guelph, ON, Canada). 


\section{Semi-quantification using external calibration curve and internal standards}

CECs confirmed by reference standards were quantified based on 4-point external calibration curves $(1,5,50,200 \mu \mathrm{g} / \mathrm{L})$ on the UHPLC-qTOF, using the same instrumental methods as were used for analyzing the marine water samples. The concentration range of the calibration curve was based on the estimated concentration range in marine water $(0-100 \mathrm{ng} / \mathrm{L})$ and the $1000 \mathrm{x}$ concentration from solid phase extraction processing, resulting in an in-vial concentrations of 0 $100 \mu \mathrm{g} / \mathrm{L}$. Concentrations were first measured based on the external calibration curves. Next, for each internal standard (IS, 11 compounds in ESI+ and 8 compounds in ESI-, Table S2) spiked in the samples and the calibrants, IS response ratios were calculated based on their peak areas (Equation 1), and the median response ratio was calculated amongst all of the IS. Then, the normalized concentrations were calculated by dividing measured concentrations with median response ratio (Equation 2), to account for differences between calibrants and samples.

$$
\text { IS response ratio }=\frac{\text { IS peak area in sample }}{\text { IS peak area in calibration curve }}(1)
$$

normalized concentration $=$ measured concentration $/$ median IS response ratio (2)

Although the loss during sample extraction processes was not be taken into consideration, the variation from instrumental sensitivity and matrix effects were taken into account by the IS correction method. 


\section{Supporting Information Tables (Table S4 and S5 in a separate Excel file)}

Table S1. Information for marine water sampling sites and date sampled.

\begin{tabular}{|c|c|c|c|c|c|c|c|c|}
\hline No. & Site Name & Latitude & Longitude & Apr & May & Jun & Aug & Oct \\
\hline 1 & Port Townsend Water Street & 48.10917 & -122.76630 & & $\sqrt{ }$ & $\sqrt{ }$ & $\sqrt{ }$ & $\sqrt{ }$ \\
\hline 2 & Point No Point & 47.90860 & -122.52670 & $\sqrt{ }$ & $\sqrt{ }$ & $\sqrt{ }$ & $\sqrt{ }$ & $\sqrt{ }$ \\
\hline 3 & Silverdale, Dyes Inlet & 47.64279 & -122.69671 & $\sqrt{ }$ & $\sqrt{ }$ & $\sqrt{ }$ & $\sqrt{ }$ & $\sqrt{ }$ \\
\hline 4 & Hood Canal Holly & 47.57058 & -122.97154 & $\sqrt{ }$ & $\sqrt{ }$ & $\sqrt{ }$ & $\sqrt{ }$ & $\sqrt{ }$ \\
\hline 5 & Evergreen Rotary Park & 47.57617 & -122.62899 & $\sqrt{ }$ & $\sqrt{ }$ & $\sqrt{ }$ & $\sqrt{ }$ & $\sqrt{ }$ \\
\hline 6 & Commencement Bay Skookum & 47.28931 & -122.40957 & & $\sqrt{ }$ & $\sqrt{ }$ & $\sqrt{ }$ & $\sqrt{ }$ \\
\hline 7 & Hammersley Inlet-Arcadia Point & 47.19903 & -122.93953 & $\sqrt{ }$ & $\sqrt{ }$ & $\sqrt{ }$ & $\sqrt{ }$ & $\sqrt{ }$ \\
\hline 8 & Budd Inlet, West Bay & 47.06035 & -122.91503 & $\sqrt{ }$ & $\sqrt{ }$ & $\sqrt{ }$ & $\sqrt{ }$ & $\sqrt{ }$ \\
\hline 9 & Saltar's Point & 47.16998 & -122.61066 & $\sqrt{ }$ & $\sqrt{ }$ & $\sqrt{ }$ & $\sqrt{ }$ & $\sqrt{ }$ \\
\hline 10 & Salmon Beach & 47.29181 & -122.52806 & $\sqrt{ }$ & $\sqrt{ }$ & $\sqrt{ }$ & $\sqrt{ }$ & $\sqrt{ }$ \\
\hline 11 & Commencement Bay Thea Foss & 47.25919 & -122.43469 & & $\sqrt{ }$ & $\sqrt{ }$ & $\sqrt{ }$ & $\sqrt{ }$ \\
\hline 12 & Edmonds Ferry & 47.81418 & -122.38215 & & $\sqrt{ }$ & $\sqrt{ }$ & $\sqrt{ }$ & $\sqrt{ }$ \\
\hline 13 & Everett Boat Launch & 48.00523 & -122.22237 & & $\sqrt{ }$ & $\sqrt{ }$ & $\sqrt{ }$ & $\sqrt{ }$ \\
\hline 14 & Salmon Bay, Commodore Park & 47.66630 & -122.40180 & & $\sqrt{ }$ & $\sqrt{ }$ & $\sqrt{ }$ & $\sqrt{ }$ \\
\hline 15 & Smith Cove, Terminal 91 & 47.63237 & -122.37868 & & $\sqrt{ }$ & $\sqrt{ }$ & $\sqrt{ }$ & $\sqrt{ }$ \\
\hline 16 & West Point South & 47.65930 & -122.43348 & & $\sqrt{ }$ & $\sqrt{ }$ & $\sqrt{ }$ & $\sqrt{ }$ \\
\hline 17 & Joe Block Park & 47.58491 & -122.36708 & & & $\sqrt{ }$ & $\sqrt{ }$ & $\sqrt{ }$ \\
\hline 18 & Jack Perry Memorial Park & 47.58890 & -122.34869 & & & $\sqrt{ }$ & $\sqrt{ }$ & $\sqrt{ }$ \\
\hline
\end{tabular}

April $17^{\text {th }}$ accumulated precipitation $0.5-0.7$ inch

Oct $30^{\text {th. }}$ accumulated precipitation $0.9-1.2$ inch (data source: weather.gov) 
Table S2. Isotope-labelled internal standard mixture for qTOF analysis.

\begin{tabular}{llllll}
\hline Compound & Formula & RT & $\begin{array}{l}\text { ESI } \\
\text { polarity }\end{array}$ & $\begin{array}{l}\text { Conc. in } \\
\text { injection vial } \\
\text { (ng/mL) }\end{array}$ & $\begin{array}{l}\text { Range of Matrix } \\
\text { Effects (\%) }\end{array}$ \\
\hline Carbamazepine-d10 & $\mathrm{C}_{15} \mathrm{H}_{2} \mathrm{D}_{10} \mathrm{~N}_{2} \mathrm{O}$ & 6.32 & + & 25 & $77-120$ \\
Caffeine-13C3 & ${ }^{13} \mathrm{C}_{3} \mathrm{C}_{5} \mathrm{H}_{10} \mathrm{~N}_{4} \mathrm{O}_{2}$ & 3.98 & + & 50 & $93-157$ \\
Cotinine-d3 & $\mathrm{C}_{10} \mathrm{H}_{9} \mathrm{D}_{3} \mathrm{~N}_{2} \mathrm{O}$ & 3.39 & + & 100 & $67-86$ \\
Sulfamethoxazole-d4 & $\mathrm{C}_{10} \mathrm{H}_{7} \mathrm{D}_{4} \mathrm{~N}_{3} \mathrm{O}_{3} \mathrm{~S}$ & 4.13 &,+- & 100 & $69-133$ \\
Vanillin-d3 & $\mathrm{C}_{8} \mathrm{H}_{5} \mathrm{D}_{3} \mathrm{O}_{3}$ & 4.29 & - & 100 & $87-149$ \\
Sulfadimethoxine-d6 & $\mathrm{C}_{12} \mathrm{H}_{8} \mathrm{D}_{6} \mathrm{~N}_{4} \mathrm{O}_{4} \mathrm{~S}$ & 4.86 &,+- & 100 & $52-127$ \\
5-methyl-1H-benzotriazole-d6 & $\mathrm{C}_{7} \mathrm{HD}_{6} \mathrm{~N}_{3}$ & 4.93 &,+- & 100 & $67-91$ \\
Ethyl paraben-d4 & $\mathrm{C}_{9} \mathrm{H}_{6} \mathrm{D}_{4} \mathrm{O}_{3}$ & 5.92 & - & 100 & $109-170$ \\
DEET-d7 & $\mathrm{C}_{12} \mathrm{H}_{10} \mathrm{D}_{7} \mathrm{NO}$ & 7.15 & + & 100 & $62-89$ \\
Atrazine-d5 & $\mathrm{C}_{8} \mathrm{H}_{9} \mathrm{D}_{5} \mathrm{ClN}_{5}$ & 6.98 &,+- & 100 & $51-77$ \\
Prometon-d3 & $\mathrm{C}_{10} \mathrm{H}_{16} \mathrm{D}_{3} \mathrm{~N}_{5} \mathrm{O}$ & 7.81 & + & 100 & $51-74$ \\
Ibuprofen-d3 & $\mathrm{C}_{13} \mathrm{H}_{15} \mathrm{D}_{3} \mathrm{O}_{2}$ & 8.93 & - & 100 & $66-109$ \\
Bis(2-ethylhexyl)phthalate-d4 & $\mathrm{C}_{24} \mathrm{H}_{34} \mathrm{D}_{4} \mathrm{O}_{4}$ & 16.73 & + & 100 & $58-92$ \\
Theobromine-d3 & $\mathrm{C}_{7} \mathrm{H}_{2} \mathrm{D}_{6} \mathrm{~N}_{4} \mathrm{O}_{2}$ & 2.99 & + & 200 & $54-87$ \\
Nicotine-d3 & $\mathrm{C}_{10} \mathrm{H}_{11} \mathrm{D}_{3} \mathrm{~N}_{2}$ & 1.88 & + & 500 & $76-131$ \\
Propyl paraben-d4 & $\mathrm{C}_{10} \mathrm{H}_{8} \mathrm{D}_{4} \mathrm{O}_{3}$ & 7.07 & - & 500 & $97-161$ \\
\hline
\end{tabular}

$\mathrm{a}: \mathrm{RT}=$ retention time, unit: $\min$.

Table S3. Parameters for XCMS Online. Positive and negative mode shared same parameters except for adduct.

\begin{tabular}{lll}
\hline Parameter Category & Parameter & Setting \\
\hline \multirow{4}{*}{ Feature detection } & method & centWave \\
& mzdiff & 0.01 \\
& S/N threshold & 6 \\
& noise filter & 1000 \\
\hline Retention time & method & obiWarp \\
correction & profStep $(m / z)$ & 0.1 \\
\hline \multirow{3}{*}{ Alignment } & bin width $(\mathrm{s})$ & 5 \\
& minfrac & 0.65 \\
& mzwid & 0.015 \\
Statistics & test & $\mathrm{Welch} \mathrm{T}$ test \\
& $p$ value threshold & 0.05 \\
& fold change threshold & 5 \\
\hline \multirow{3}{*}{ Annotation } & ppm & 10 \\
& $m / z$ absolute error & 0.015 \\
& & {$[\mathrm{M}+\mathrm{H}],[\mathrm{M}+\mathrm{Na}]$,} \\
& adduct & {$[\mathrm{M}+\mathrm{NH} 4],[\mathrm{M}-\mathrm{H}]$} \\
\hline
\end{tabular}


Table S6. Predicted "dye" concentration from the Salish Sea Model, describing the distribution, resulting from transport of wastewater effluent from 99 unique WWTP outfalls throughout Puget Sound (mean \pm standard deviation). The concentration of all WWTP effluent was 100.

\begin{tabular}{|c|c|c|c|c|c|c|c|c|}
\hline \multirow{2}{*}{$\frac{\text { Site No. } \text { Site Name }}{4 \text { HoodCanal }}$} & \multicolumn{3}{|c|}{ May } & \multicolumn{3}{|c|}{ June } & \multicolumn{2}{|c|}{ August } \\
\hline & 0.06515 & \pm & 0.00173 & 0.06650 & \pm & 0.00251 & $0.06261 \pm$ & 0.00212 \\
\hline 5 EvergreenRPk & 0.22197 & \pm & 0.03161 & 0.21370 & \pm & 0.03522 & $0.28917 \pm$ & 0.06196 \\
\hline 8 BuddInlet & 0.53766 & \pm & 0.13805 & 0.63766 & \pm & 0.12424 & $0.73726 \pm$ & 0.13873 \\
\hline 9 SaltarsPoint & 0.25351 & \pm & 0.04964 & 0.24373 & \pm & 0.04720 & $0.20957 \pm$ & 0.03695 \\
\hline 11 CommBayTheaFoss & 0.20374 & \pm & 0.01218 & 0.18944 & \pm & 0.00430 & $0.20060 \pm$ & 0.01115 \\
\hline 12 EdmondsFerry & 0.15112 & \pm & 0.00728 & 0.14909 & \pm & 0.00579 & $0.13920 \pm$ & 0.00289 \\
\hline 13 EverettBoatLaunch & 0.15342 & \pm & 0.01429 & 0.16812 & \pm & 0.01210 & $0.14782 \pm$ & 0.00685 \\
\hline 14 SalmonBayCPk & 0.04041 & \pm & 0.01176 & 0.05731 & \pm & 0.01635 & $0.09332 \pm$ & 0.00703 \\
\hline 15 SmithCove & 0.16226 & \pm & 0.01017 & 0.15680 & \pm & 0.00631 & $0.14526 \pm$ & 0.00393 \\
\hline 16 WestPointSouth & 0.16500 & \pm & 0.00851 & 0.16211 & \pm & 0.00691 & $0.14453 \pm$ & 0.00309 \\
\hline 18 JackPerryMPk & 0.07627 & \pm & 0.01148 & 0.08622 & \pm & 0.01082 & $0.12631 \pm$ & 0.00604 \\
\hline
\end{tabular}

Table S7. Numbers of non-target features at each site/date (passed through replicate filters and blank subtraction).

\begin{tabular}{|c|c|c|c|c|c|c|c|c|c|c|c|c|c|c|}
\hline $\begin{array}{l}\text { Site } \\
\text { No. }\end{array}$ & $\begin{array}{l}\text { Apr } \\
\text { pos }\end{array}$ & $\begin{array}{l}\text { Apr } \\
\text { neg }\end{array}$ & $\begin{array}{l}\text { May } \\
\text { pos }\end{array}$ & $\begin{array}{l}\text { May } \\
\text { neg }\end{array}$ & $\begin{array}{l}\text { Jun } \\
\text { pos }\end{array}$ & $\begin{array}{l}\text { Jun } \\
\text { neg }\end{array}$ & $\begin{array}{l}\text { Aug } \\
\text { pos }\end{array}$ & $\begin{array}{l}\text { Aug } \\
\text { neg }\end{array}$ & $\begin{array}{l}\text { Oct } \\
\text { pos }\end{array}$ & $\begin{array}{l}\text { Oct } \\
\text { neg }\end{array}$ & $\begin{array}{l}\text { Sum } \\
\text { Pos }\end{array}$ & $\begin{array}{l}\text { Sum } \\
\text { Neg }\end{array}$ & Sum & $\begin{array}{l}\text { Average No. } \\
\text { of features }\end{array}$ \\
\hline 1 & & & 335 & 252 & 625 & 308 & 604 & 332 & 606 & 121 & 2170 & 1013 & 3183 & 796 \\
\hline 2 & 231 & 141 & 388 & 226 & 442 & 319 & 473 & 289 & 472 & 101 & 2006 & 1076 & 3082 & 616 \\
\hline 3 & 315 & 210 & 337 & 199 & 449 & 228 & 733 & 415 & 597 & 136 & 2431 & 1188 & 3619 & 724 \\
\hline 4 & 268 & 135 & 265 & 175 & 350 & 192 & 526 & 373 & 251 & 98 & 1660 & 973 & 2633 & 527 \\
\hline 5 & 258 & 111 & 327 & 196 & 503 & 290 & 697 & 412 & 445 & 117 & 2230 & 1126 & 3356 & 671 \\
\hline 6 & & & 405 & 213 & 420 & 209 & 533 & 368 & 480 & 131 & 1838 & 921 & 2759 & 690 \\
\hline 7 & 343 & 264 & 468 & 312 & 635 & 291 & 611 & 349 & 562 & 151 & 2619 & 1367 & 3986 & 797 \\
\hline 8 & 271 & 139 & 133 & 60 & 544 & 269 & 586 & 357 & 490 & 131 & 2024 & 956 & 2980 & 596 \\
\hline 9 & 335 & 204 & 334 & 170 & 349 & 153 & 669 & 381 & 436 & 129 & 2123 & 1037 & 3160 & 632 \\
\hline 10 & 357 & 285 & 353 & 158 & 381 & 192 & 342 & 271 & 385 & 114 & 1818 & 1020 & 2838 & 568 \\
\hline 11 & & & 417 & 257 & 619 & 308 & 628 & 366 & 535 & 134 & 2199 & 1065 & 3264 & 816 \\
\hline 12 & & & 377 & 251 & 509 & 244 & 706 & 412 & 210 & 55 & 1802 & 962 & 2764 & 691 \\
\hline 13 & & & 68 & 61 & 318 & 147 & 248 & 136 & 351 & 69 & 985 & 413 & 1398 & 350 \\
\hline 14 & & & 255 & 151 & 379 & 191 & 452 & 270 & 291 & 57 & 1377 & 669 & 2046 & 512 \\
\hline 15 & & & 437 & 316 & 552 & 241 & 642 & 284 & 326 & 74 & 1957 & 915 & 2872 & 718 \\
\hline 16 & & & 496 & 335 & 470 & 339 & 628 & 368 & 303 & 97 & 1897 & 1139 & 3036 & 759 \\
\hline 17 & & & & & 347 & 218 & 417 & 266 & 148 & 47 & 912 & 531 & 1443 & 481 \\
\hline 18 & & & & & 289 & 120 & 311 & 192 & 261 & 60 & 861 & 372 & 1233 & 411 \\
\hline
\end{tabular}


Table S8. Summary of linear regression between the most prevalent CECs and the predicted dye concentration from the Salish Sea Model. The dye represents the combined contributions and transport of WWTP effluent from 99 outfalls throughout Puget Sound.

\begin{tabular}{lcccccc}
\hline & \multicolumn{2}{c}{ May } & \multicolumn{2}{c}{ June } & \multicolumn{2}{c}{ August } \\
\hline & $\mathrm{R}^{2}$ & $p^{a}$ & $\mathrm{R}$ & $p$ & $\mathrm{R}^{2}$ & $p$ \\
\hline Lamotrigine & 0.5 & 0.085 & 0.73 & $\mathbf{0 . 0 2 4}^{\mathrm{b}}$ & 0.65 & $\mathbf{0 . 0 1 8}$ \\
Sucralose & 0.25 & 0.25 & 0.82 & $\mathbf{0 . 0 4 7}$ & 0.7 & $\mathbf{0 . 0 0 5 1}$ \\
2-Hydroxyquinoline & 0.078 & 0.77 & 0.0035 & 0.99 & 0.13 & 0.36 \\
4-Nitrophenol & 0.071 & 0.74 & 0.41 & 0.18 & 0.0079 & 0.91 \\
\hline
\end{tabular}

a. $p$ values were calculated from $95 \%$ quantile of a 10,000-replicate bootstrap

b. bold figures indicate statistically significant results $(\alpha=0.05)$ 
Supporting Information Figures

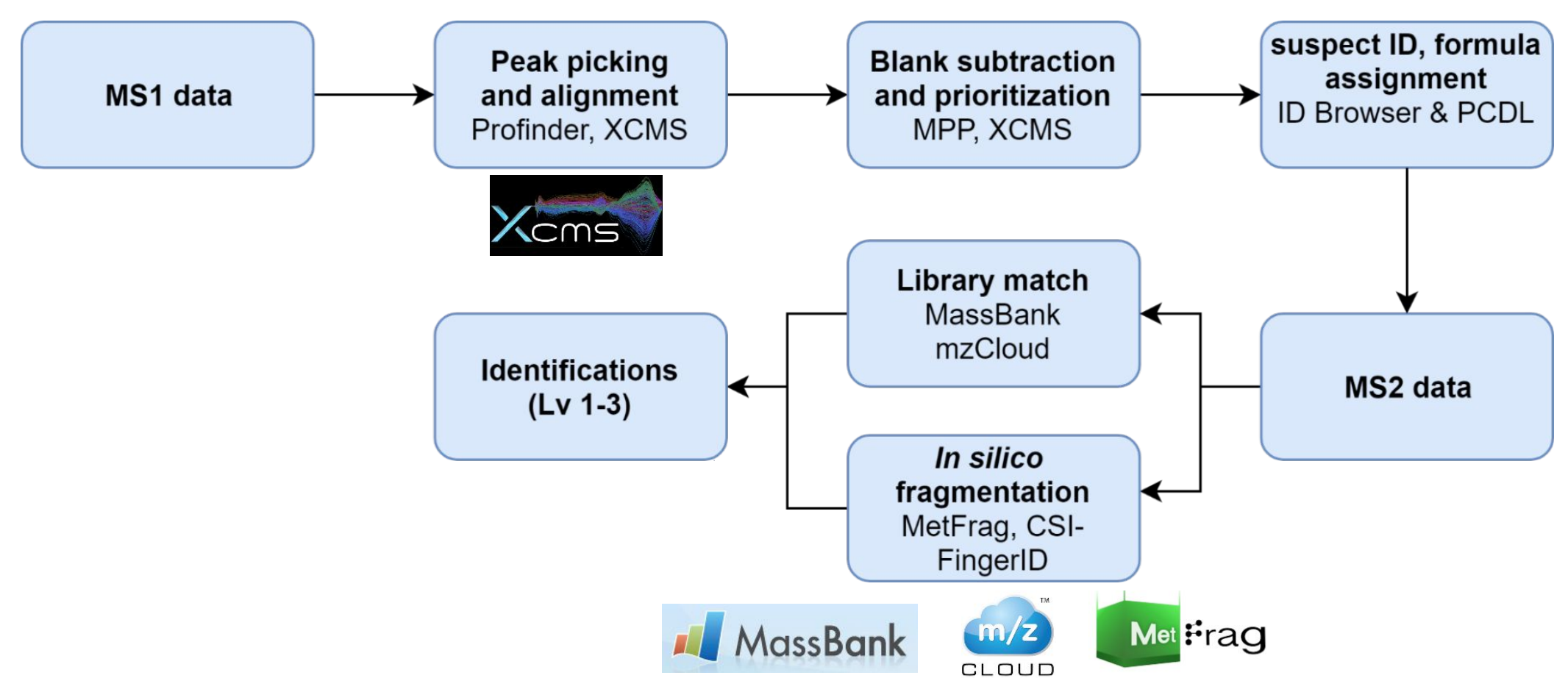

Figure S1. The workflow for data prioritization and identification. MPP = Mass Profiler Professional, PCDL $=$ Personal Compound Database and Library. 


$\begin{array}{lll}\begin{array}{l}\text { Peak picking } \\ \text { (peak height }>5000\end{array} & \text { Replicate filter } & \text { Blank subtraction } \\ \text { or area }>50000) & \text { (3 of } 3 \text { analytical } & (5 x>\text { solvent blank, } \\ & \text { replicates) } & \text { method blank, field } \\ \text { blank) }\end{array}$
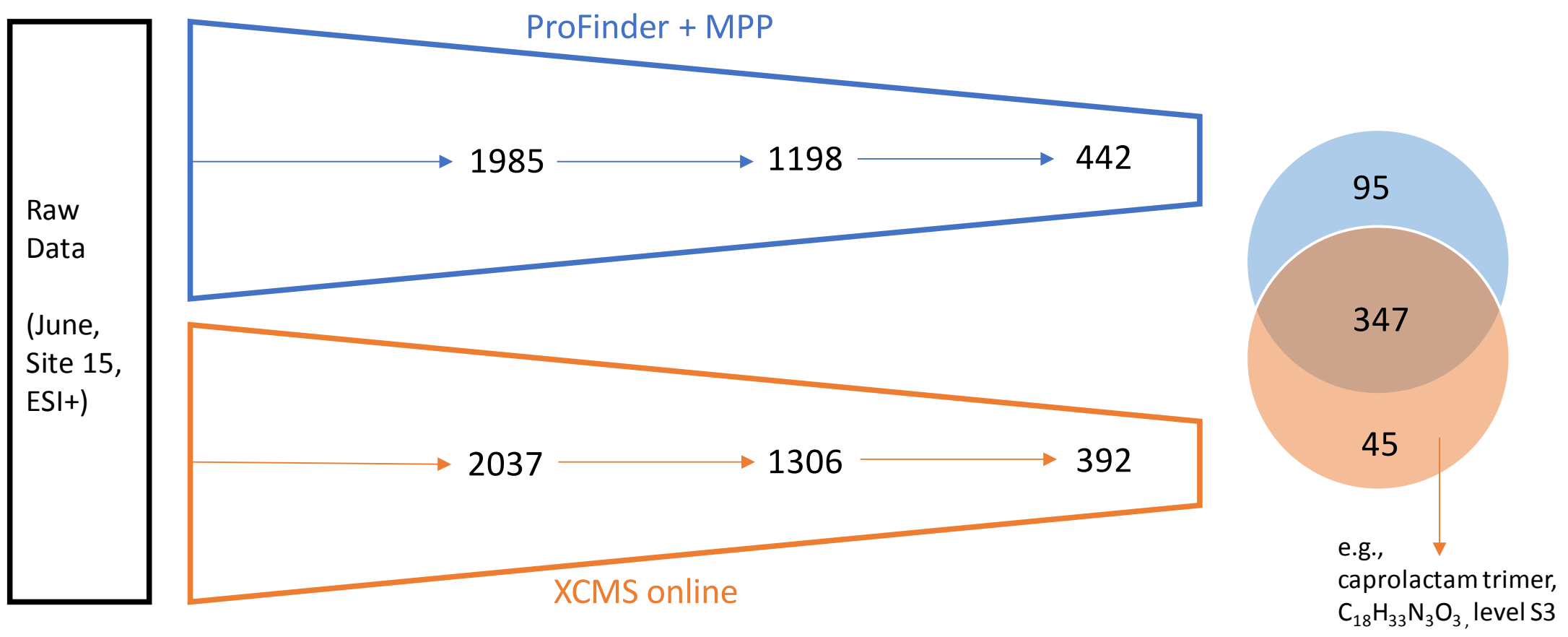

Figure S2. Comparison of the two workflows (Profinder + MPP and XCMS online) for feature detection and prioritization. The example data was from Site 15, June 2018, in ESI+ mode. The numbers represent the amounts of features. 


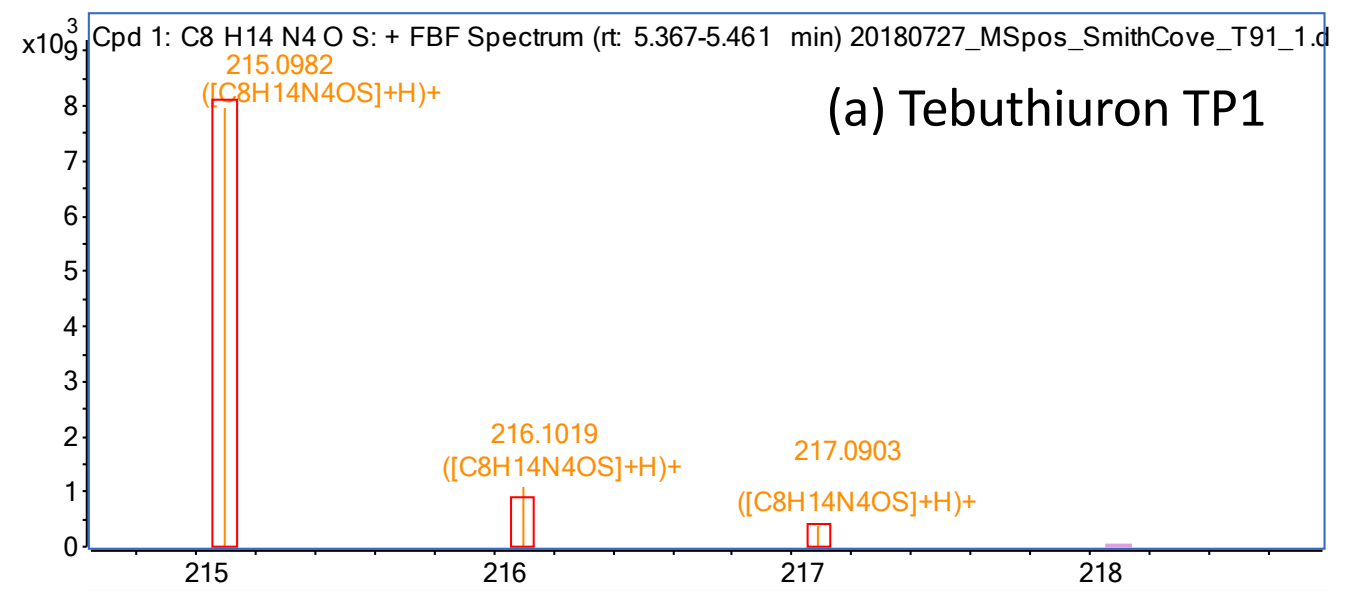

Subtract
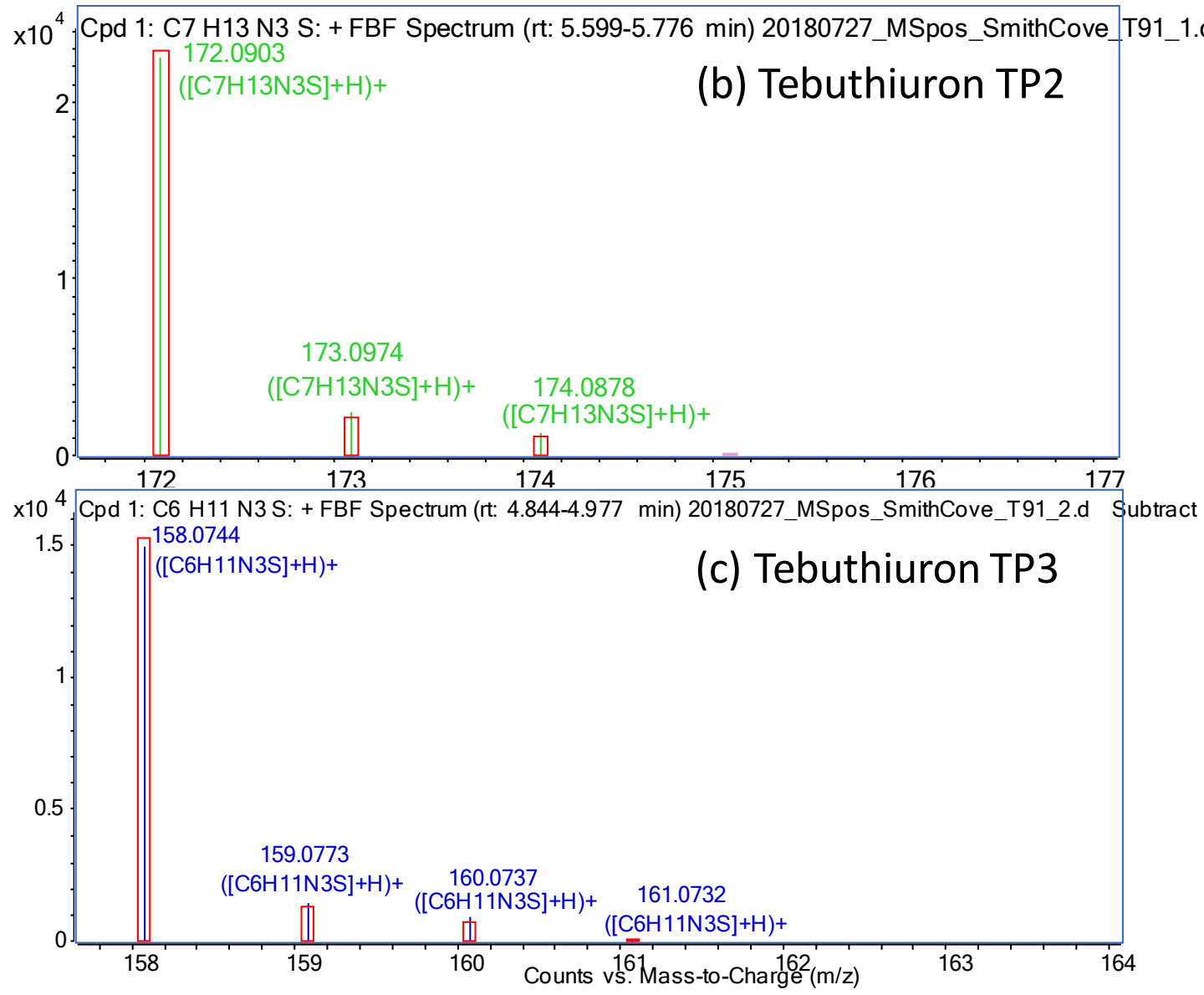

Figure S3. Isotope patterns of tebuthiuron TPs and chlorothalonil TPs: (a) tebuthiuron TP1; (b) tebuthiuron TP2; (c) tebuthiuron TP3; (d) 4-hydroxy-chlorothalonil; (e) 1-amide-4-hydroxychlorothalonil. The red boxes represent theoretical isotope patterns calculated by Agilent Qualitative Analysis, and the bars are measured mass spectra. 

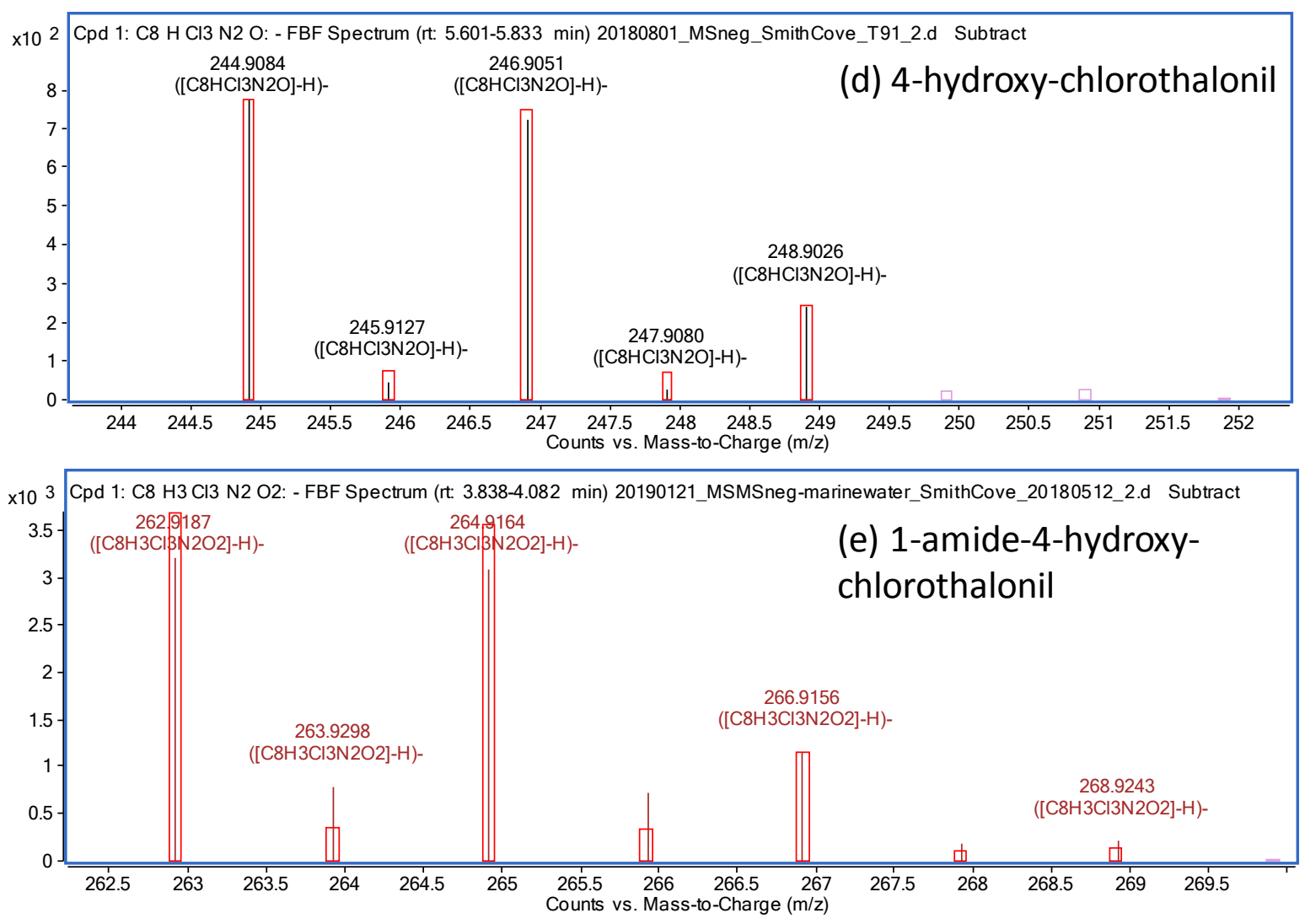

Figure S3, continued. 
(a) 4-((4-Isopropoxyphenyl)sulfonyl)phenol $\left(\mathrm{C}_{15} \mathrm{H}_{16} \mathrm{O}_{4} \mathrm{~S}\right)$

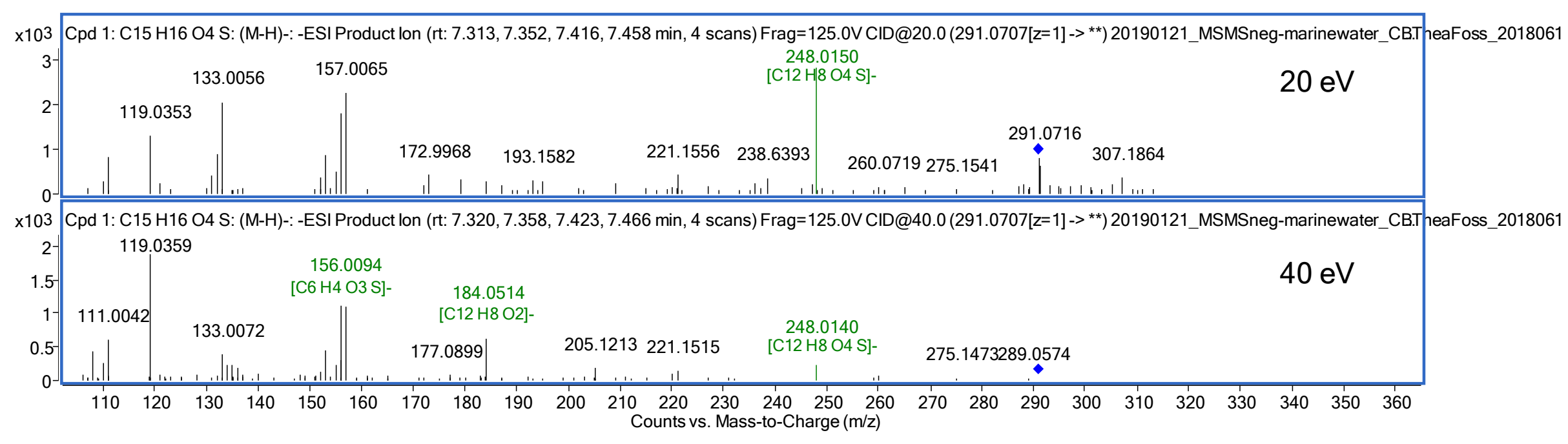

\section{(b) 1-Amide-4-hydroxy-chlorothalonil $\left(\mathrm{C}_{8} \mathrm{H}_{3} \mathrm{Cl}_{3} \mathrm{~N}_{2} \mathrm{O}_{2}\right)$}

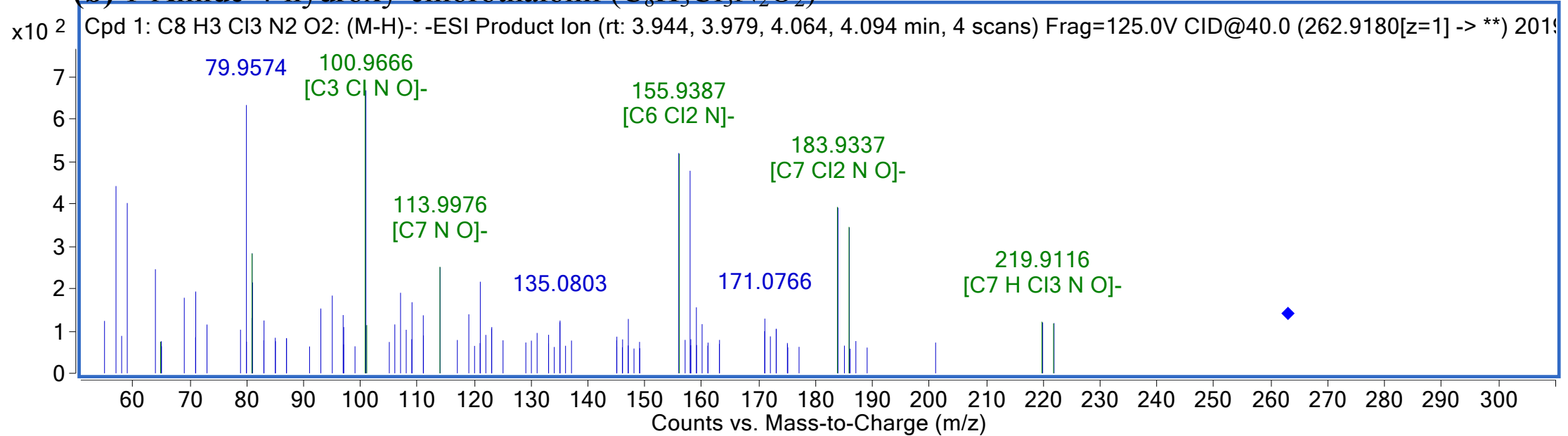

Figure S4. MS/MS spectra for level S3 tentatively identified contaminants. The blue diamonds in the spectra indicate the parent ions. (a): 4-((4-Isopropoxyphenyl)sulfonyl)phenol featured by a fragment related to bisphenol $\mathrm{S}\left(\mathrm{C}_{12} \mathrm{H}_{8} \mathrm{O}_{4} \mathrm{~S}\right)$. (b):1-Amide-4-hydroxychlorothalonil lost a $\mathrm{CONH}_{2}$ (amide group) and a $\mathrm{Cl}$ atom. 
(c) Tolyl diphenyl phosphate $\left(\mathrm{C}_{19} \mathrm{H}_{17} \mathrm{O}_{4} \mathrm{P}\right)$

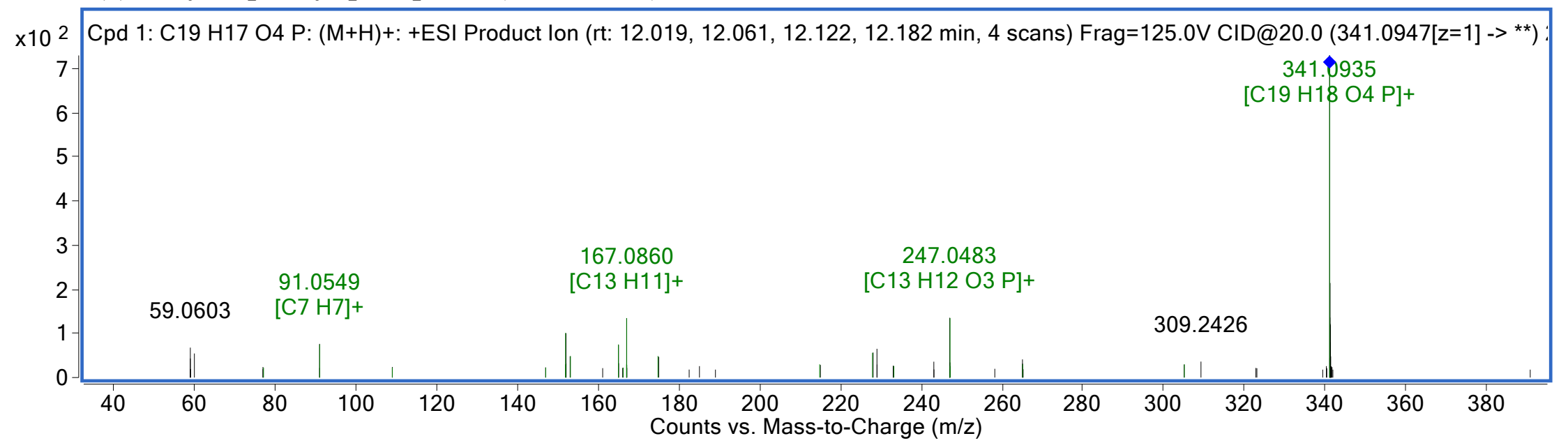

(d) Phenyl di(p-tolyl) phosphate $\left(\mathrm{C}_{20} \mathrm{H}_{19} \mathrm{O}_{4} \mathrm{P}\right)$

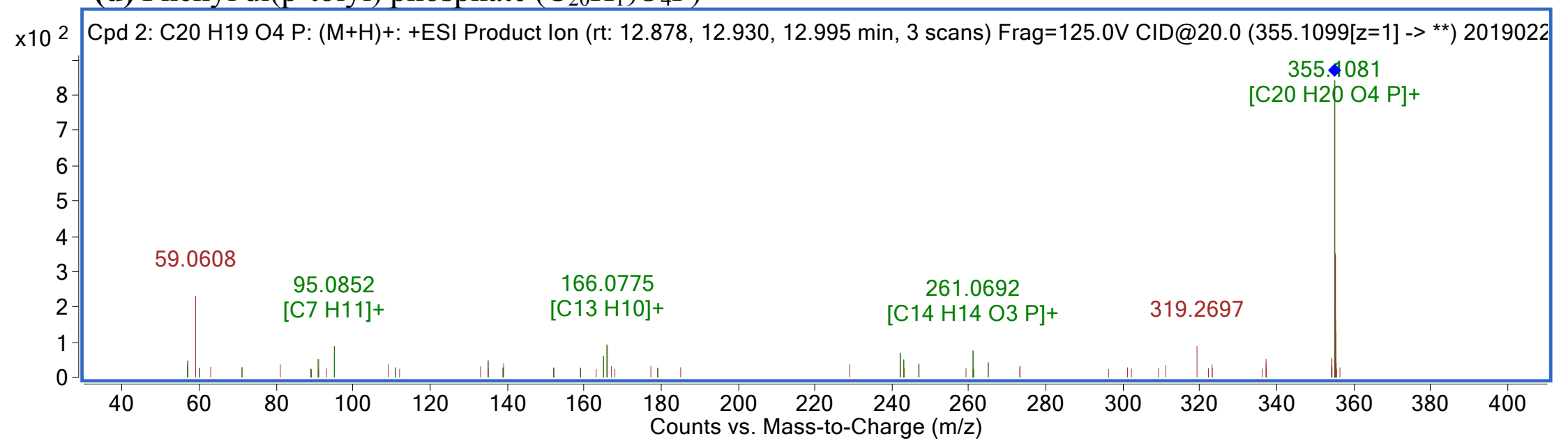

Figure S4 (continued). MS/MS spectra for level S3 tentatively identified contaminants. Both (c) tolyl diphenyl phosphate and (d) phenyl di(p-tolyl) phosphate lost C6H6O (phenol group). 
(e) Ammonium perfluorooctanesulfonate $\left(\mathrm{C}_{8} \mathrm{H}_{4} \mathrm{~F}_{17} \mathrm{NO}_{3} \mathrm{~S}\right)$

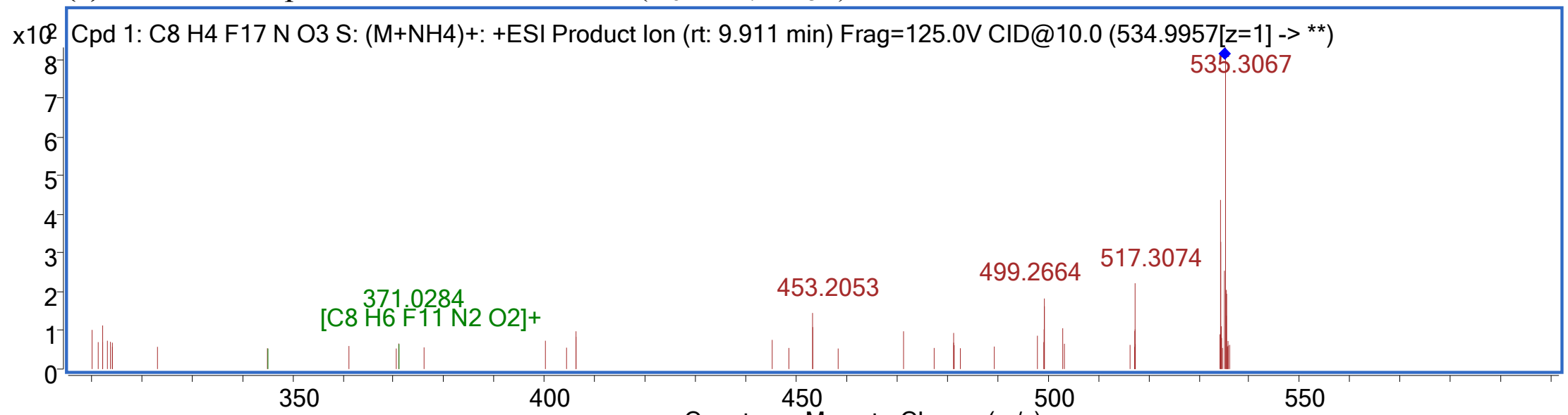

(f) N,N-dihexyl-1-decanamine $\left(\mathrm{C}_{22} \mathrm{H}_{47} \mathrm{~N}\right)$

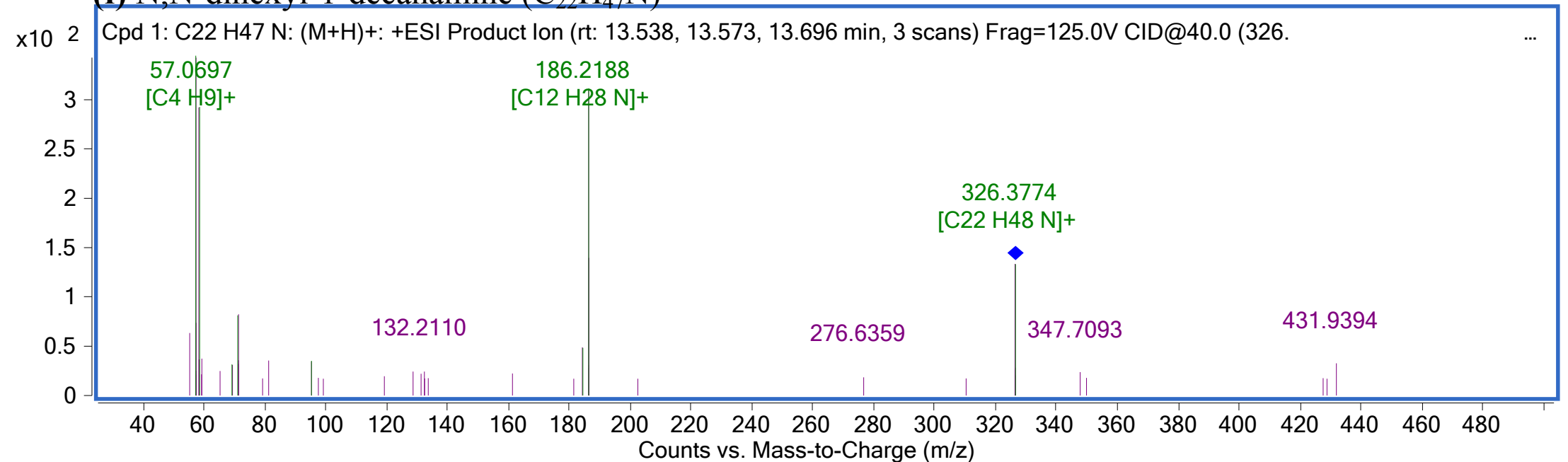

Figure S4 (continued). MS/MS spectra for level S3 tentatively identified contaminants. (e): the fragment represents PFOS $(\mathrm{m} / z$ 499) and PFOS ammonium salt $(\mathrm{m} / \mathrm{z} 517)$ can be seen. (f): the loss of $\mathrm{C}_{10} \mathrm{H}_{20}$ represent the decyl group (calculated by CSI:FingerID). 
(g) N-butyl-3-pentanamide $\left(\mathrm{C}_{9} \mathrm{H}_{19} \mathrm{NO}\right)$

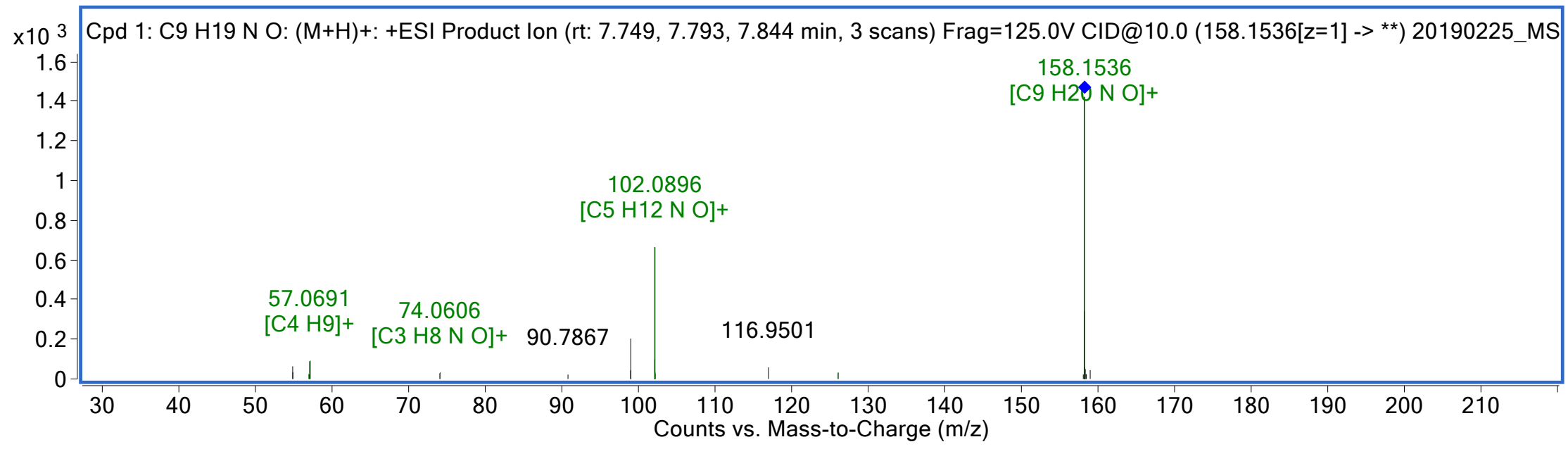

(h) Octyldimethylamine oxide $\left(\mathrm{C}_{10} \mathrm{H}_{23} \mathrm{NO}\right)$

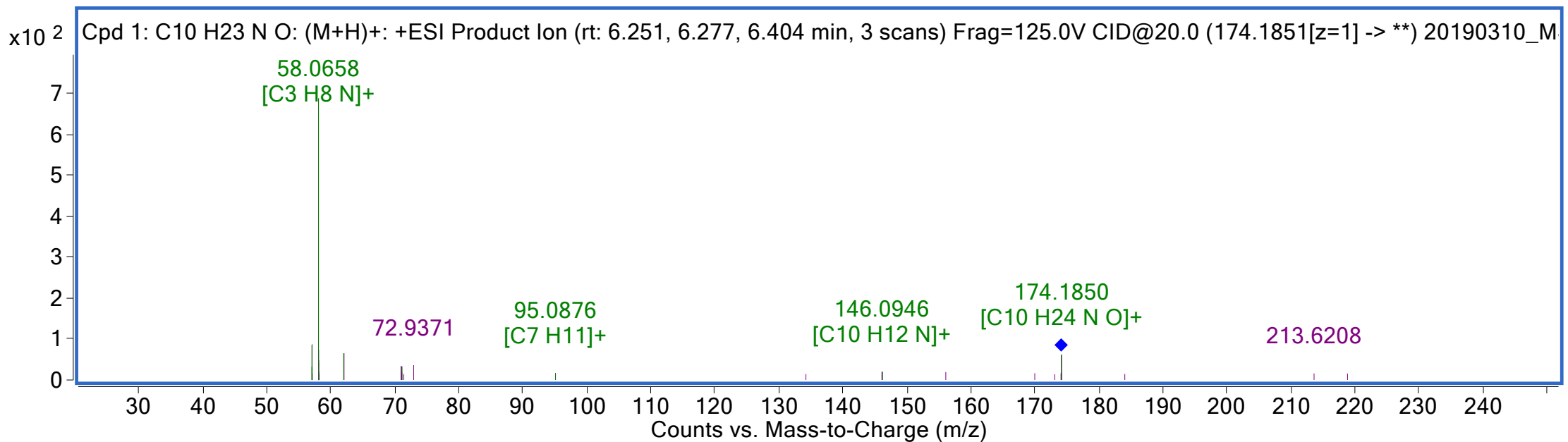

Figure S4 (continued). MS/MS spectra for level S3 tentatively identified contaminants. (g): the lost C4H8 represented the butyl group, and $\mathrm{C}_{5} \mathrm{H}_{12} \mathrm{NO}$ is the left pentanamide. (h): octyldimethylamine oxide was proposed by in silico fragmentation. 
(i) $\mathrm{N}$-[3-(dimethylamino)propyl]dodecanamide $\left(\mathrm{C}_{17} \mathrm{H}_{36} \mathrm{~N}_{2} \mathrm{O}\right)$

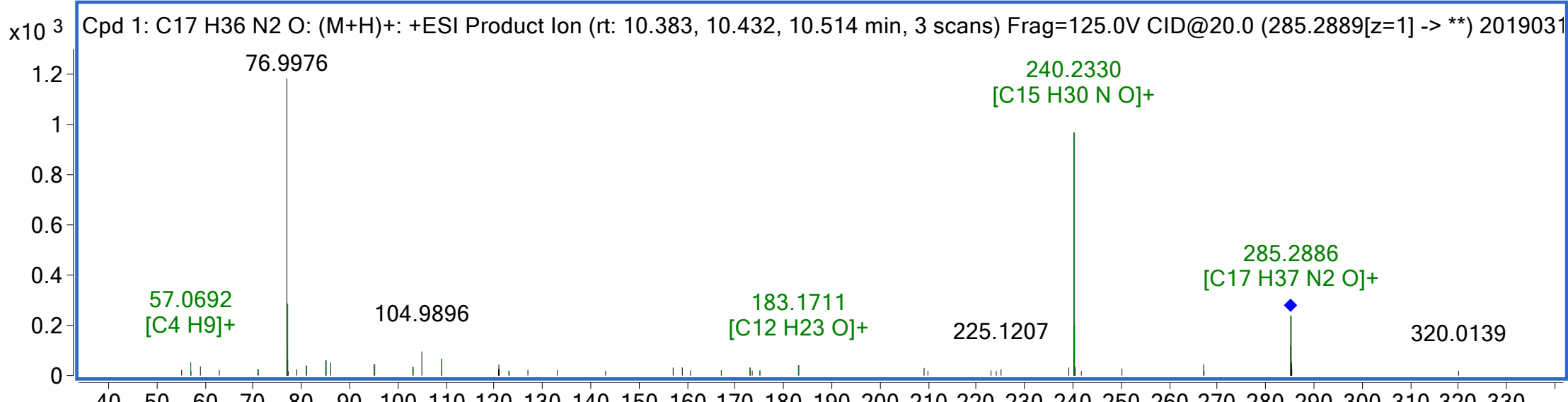

$40 \quad 50 \quad 60 \quad 70 \quad 80 \quad 90100110120130140150160170180190200210220230240250260270280290300310320330$ (j) Olivetol

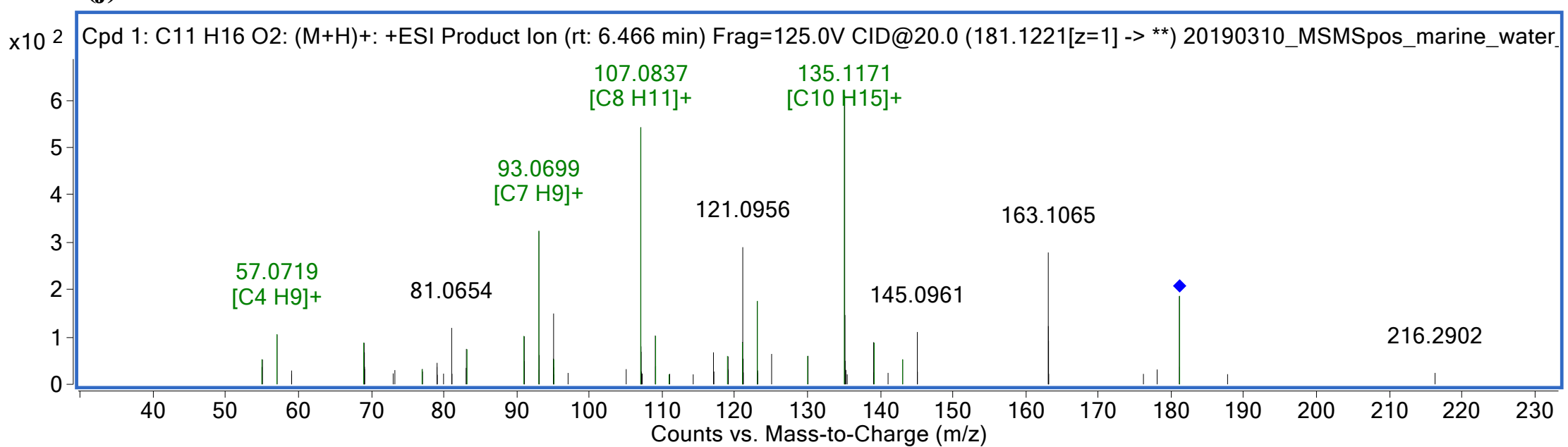

Figure S4 (continued). MS/MS spectra for level S3 tentatively identified contaminants. (i): the loss of C2H7N represented dimethylamino group. (j): olivetol was proposed by in-silico fragmentation. 


\section{(k) Caprolactam trimer}

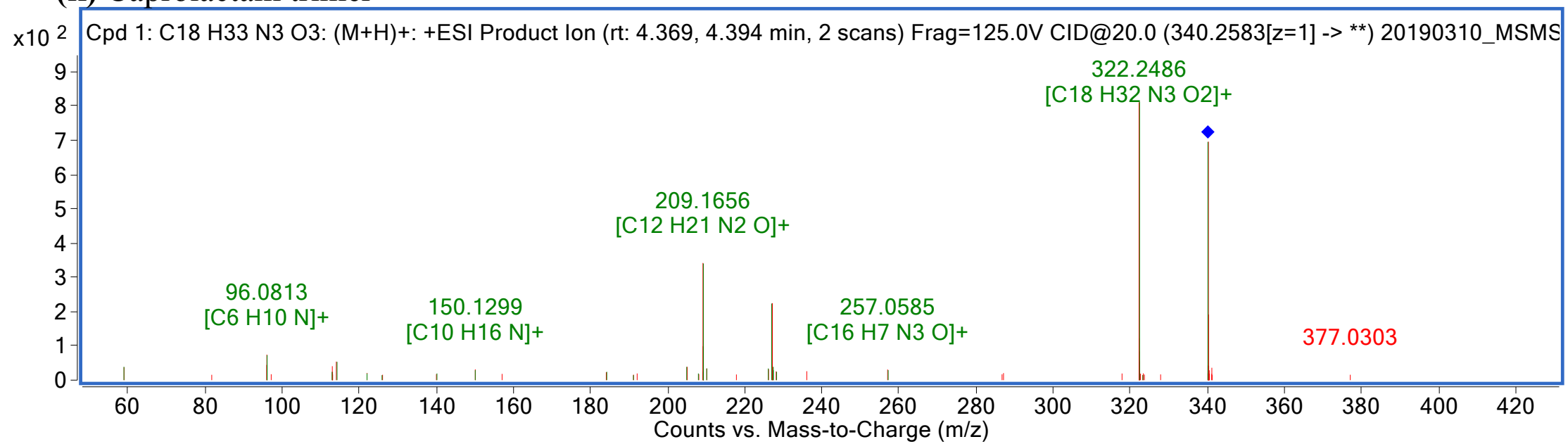

(l) 1-[5-(1-Aminoethyl)-2-methylsulfanyl-4-propylsulfanylphenyl]ethanamine

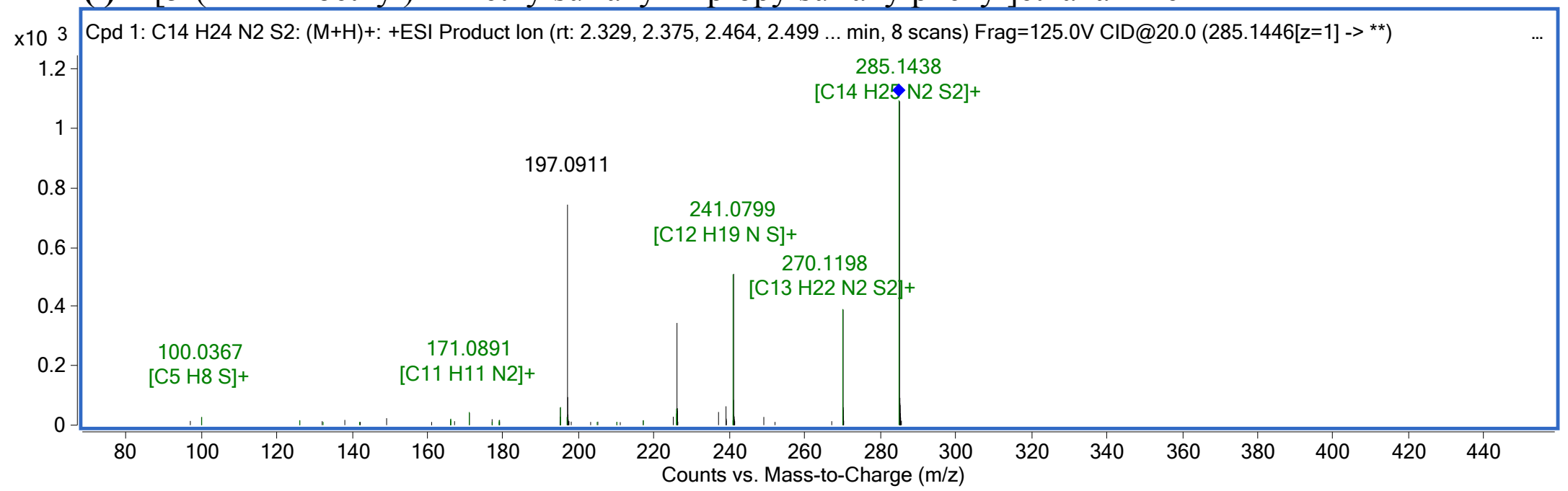

Figure S4 (continued). MS/MS spectra for level S3 tentatively identified contaminants. (k): a regular pattern of losing $\mathrm{C}_{6} \mathrm{H}_{11} \mathrm{NO}$ (caprolactam). (I): consecutive loss of $\mathrm{CH}_{3}$ and $\mathrm{C}_{2} \mathrm{H}_{6} \mathrm{~N}$ represented methyl and aminoethyl groups. 


\section{detection frequency of stormwater cpds}

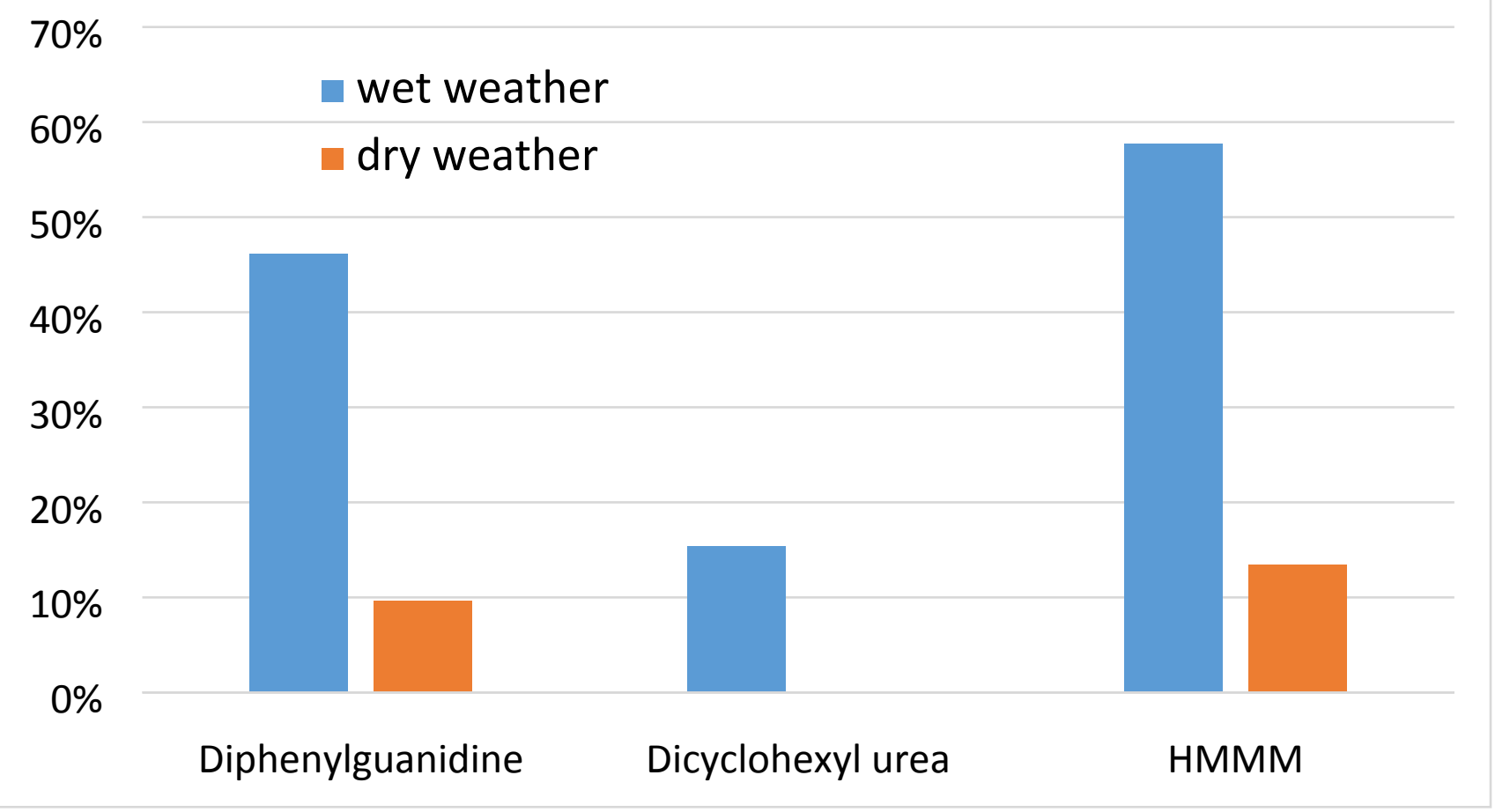

Figure S5. Detection frequencies of contaminants associated with stormwater runoff in wet weather sampling (April, October) and dry weather sampling (May, June, August). The detection frequency of stormwater-associated CECs was much higher in the wet weather sampling compared to dry weather, illustrating the potential range of impacts of stormwater in the marine nearshore. HMMM: Hexa(methoxymethyl)melamine. 


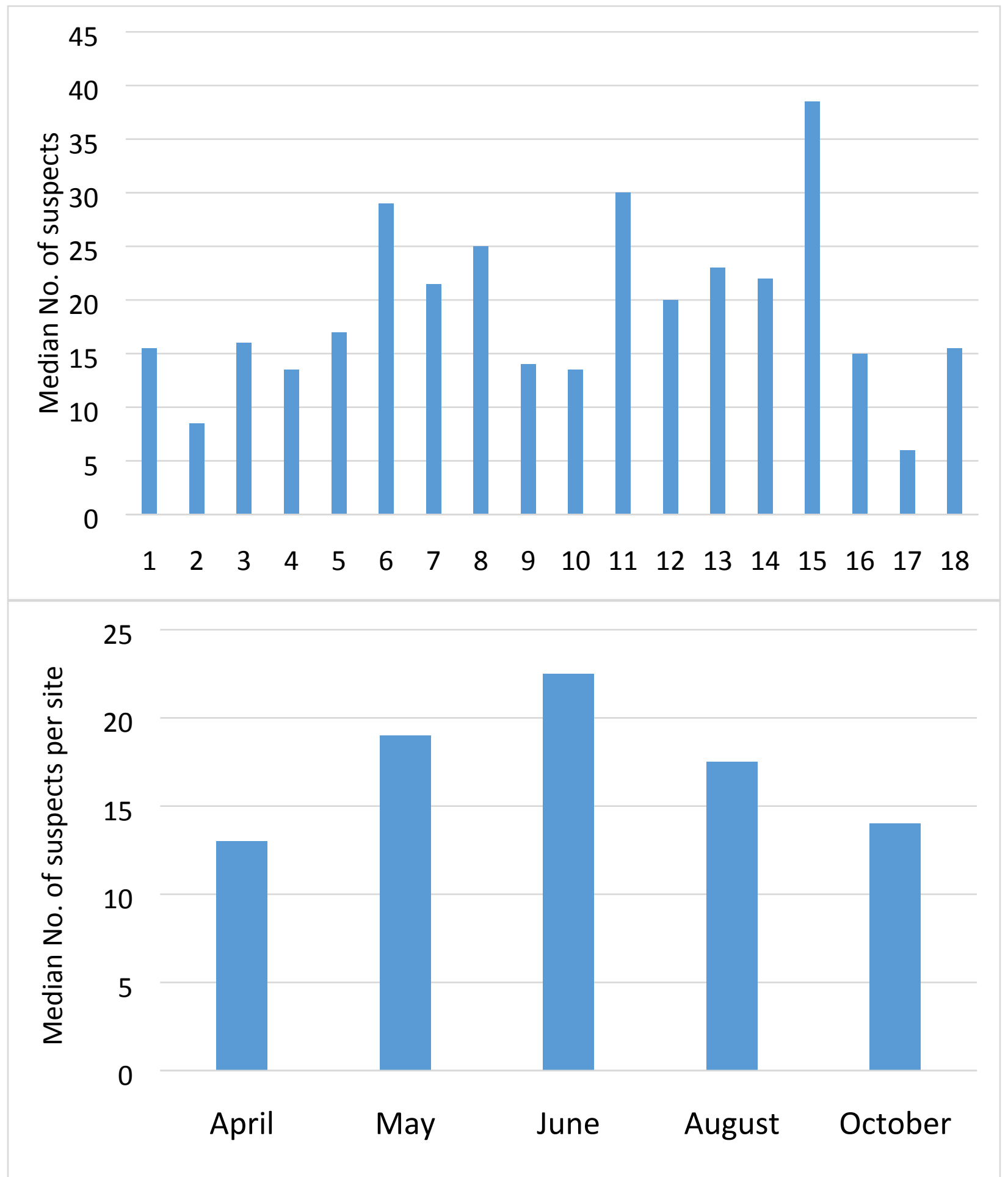

Figure S6. Temporal and spatial trends of contaminants in Puget Sound, measured by: (a) median numbers of matched suspects by sites. Site number, names, and locations are shown in Table S1 and Figure 1. (b) median numbers of matched suspects per site by dates. The suspects were from a standard compound database (CUW standards database) including wastewater-derived contaminants, stormwater contaminants, and chemicals from EPA's ToxCast library, which implies anthropogenic impacts. The database includes a total of $\sim 1100$ compounds. 


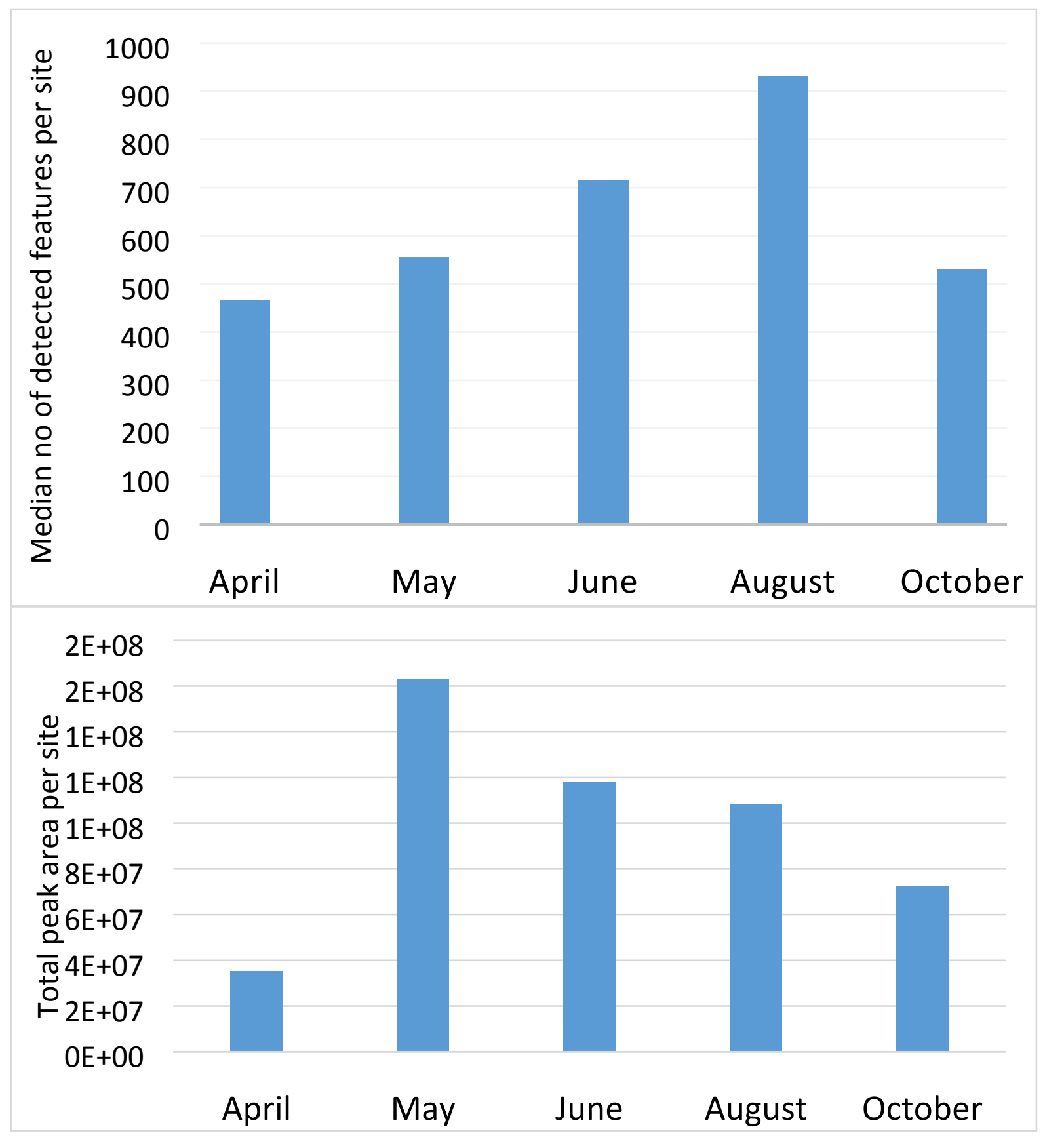

Figure S6, continued. (c) median number of features detected across all sites for each sampling event; (d) median total peak areas across all sites for each sampling event. 